\title{
Fast convergent average consensus of multiagent systems based on community detection algorithm
}

\author{
Xinlei Feng ${ }^{1,2^{*}}$ (D) and Xiaoli Yang ${ }^{3}$
}

\section{"Correspondence:}

xinlfeng@126.com

${ }^{1}$ College of Mathematics and

Information Science, Leshan Normal

University, Leshan, China

${ }^{2}$ Department of Math/Stat, Georgia

State University, Atlanta, USA

Full list of author information is

available at the end of the article

\section{黛 Springer}

\begin{abstract}
In this paper, we consider fast convergent average consensus based on community detection algorithm. Generally, we know that a small network can have a faster convergence speed than a big one at the same condition. So we divide a multiagent network into several small networks. Firstly, let every small network reach own consensus, and then entire network reach the average consensus. Based on this idea, we present FCWAC Algorithm. For the FCWAC Algorithm, we obtain the results on the average consensus of first-, second-, and high-order continuous-time multiagent systems. Finally, simulation examples illustrate our theoretical results.
\end{abstract}

Keywords: Consensus; Multiagent systems; Community detection algorithm; Continuous-time system

\section{Introduction}

In the last twenty years the distributed consensus problem of multiagent systems has attracted extensive interest of many scholars, owing to many its important practical applications, such as satellite configuration, unmanned aerial vehicle formation, and so on. The distributed consensus (we refer to it as consensus in this paper) means that agents in a communication network reach a common state by exchanging information with their neighbor agents.

In fact the consensus problem has already received attention in management science and statistics since the 1960s. Borkar and Varaiya [1] and Tsitsiklis [2] wrote some pioneering works on distributed computation of systems and control theory. Specifically, for the first time, Vicsek et al. [3] presented an analysis of the alignment problem based on a model about flocking, which was a milestone in development of the consensus theory of multiagent systems. A classical consensus model of continuous-time multiagent systems was presented by Olfati-Saber and Murray [4]. Subsequently, many scholars began to study consensus from first-order to second-order or even high-order multi-gent systems [5-17].

In 2018, the consensus problem of multiagent systems is still a hot area of research. Many scholars obtained a lot of significant results. For example, Lin et al. [18] considered a distributed consensus algorithm for the second-order multiagent systems with nonconvex velocity and control input constraints, which is introduced based on the union of the communication graphs has directed spanning trees among each time interval of cer-

(c) The Author(s) 2018. This article is distributed under the terms of the Creative Commons Attribution 4.0 International License (http://creativecommons.org/licenses/by/4.0/), which permits unrestricted use, distribution, and reproduction in any medium, provided you give appropriate credit to the original author(s) and the source, provide a link to the Creative Commons license, and indicate if changes were made. 
tain bounded length in the presence of arbitrarily bounded communication delays. Xu et al. [19] obtained the output consensus problem of discrete-time heterogeneous linear multiagent systems with switching topology and time delays by introducing a distributed predictor-based controller. Wu et al. [20] discussed the leader-following consensus problem of high-order multiagent systems via a novel distributed event-triggered communication protocol based on state estimates of neighboring agents, which can greatly reduce the communication load of multiagent networks. Zhang et al. [21] considered the distributed optimal cooperative control for continuous-time nonlinear multiagent systems (MASs) with completely unknown dynamics via adaptive dynamic programming (ADP) technology, which introduces predesigned extra compensators. A novel online iterative scheme was also proposed, which runs based on the data sampled from the augmented system and the gradient of the value function. Neural networks were employed to implement the algorithm, which yields approximated optimal consensus protocols. Rehan et al. [22] considered a distributed consensus controller design approach for one-sided Lipschitz nonlinear multiagents by employing relative state feedback. The proposed consensus control approach is less conservative for robustness against disturbances owing to its ability to handle amplitude-bounded disturbances and due to the relaxation of a balanced communication topology.

For multiagent systems, especially for those having a complicated network, improving their convergence speed is a very significant work. At present, two major approaches to improve the convergence speed to consensus are optimizing the weight of the communication topology $[5-7,12]$ and incorporating memory into the distributed algorithms [8-10], respectively. For multiagent systems, the algebraic connectivity or spectral radius of the communication topology determines their convergence speed. Therefore, we can improve the convergence speed to consensus by optimizing the weight of the communication topology. For example, Zhu et al. [12] obtained that the maximum convergence speed of multiagent systems with double-integrator dynamics can be achieved by choosing suitable gains in a periodic consensus protocol. Meanwhile, Aysal et al. [9] presented a linear local iterative protocol, which can obtain asymptotically distributed average consensus by using a linear predictor to predict subsequent states of agents. For the first time Oreshkin et al. [10] presented a theoretical demonstration, which, by adding a local predictor in distributed average consensus protocol, can effectively improve the convergence speed of multiagent systems.

However, the methods mentioned are limited when applied to high-order systems. So accelerating the consensus convergence of high-order multiagent systems is very difficult. Either it is difficult to extract the roots of a high-order equation, or there no effective methods to process a high-order equation.

However, Zhang et al. [23] presented a new community detection algorithm to accelerate consensus convergence by introducing a local modularity into the algorithm in [24-26]. The main idea of [23], which introduces the new community detection algorithm, is, firstly, dividing the entire communication topology graph of a multiagent system into several layers of connected subgraphs, where every subgraph is seen as a community and, secondly, letting all communities to reach group consensus in every layer; finally, the hierarchical groups reach the final consensus of a multiagent system.

However, the consensus in [23] has also a shortcoming; namely, it is not an average consensus. Meanwhile, the authors in [23] also only considered consensus of first-order sys- 
tems. Therefore, due to these shortcomings, we present average consensus of first-order systems based on community detection algorithm and also consider the average consensus of second- and high-order systems based on community detection algorithm.

The remainder part of this paper is organized as follows. In Sect. 2, we describe preliminary definitions and results. In Sect. 3, we present FCWAC Algorithm based on the community detection algorithm [23] and consider average consensus of first-order multiagent systems according to the FCWAC Algorithm. In Sect. 4, we consider average consensus of second- and high-order mult-agent systems according to the FCWAC Algorithm. Moreover, in Sect. 5, we present numerical examples to illustrate our theoretical results. Conclusions are drawn in Sect. 6.

\section{Preliminaries}

A directed graph (digraph) $G=(V, E)$ consists of a set of nodes $V=\{1, \ldots, n\}$ and a set of edges $E=V \times V$. A pair $(i, j)$ is an edge of $G$ if and only if $(i, j)$ is an arc from node $i$ to node $j$ of $G$. Likewise, agent $j$ is called a neighbor of agent $i$. The set of all neighbors of agent $i$ is written as $\mathcal{N}_{i}(t)$. Suppose that there exist $n$ nodes in a graph $G$. The adjacency matrix $A \in \mathbb{R}^{n \times n}$ of $G$ is defined as $a_{i j}=1$ if $(i, j) \in E$ and $a_{i j}=0$ otherwise. If a graph has the property that $(i, j) \in E$ implies $(j, i) \in E$, then it is said to be undirected. The Laplacian matrix $L \in \mathbb{R}^{n \times n}$ is defined as $l_{i i}=\sum_{j \neq i} a_{i j}$ and $l_{i j}=-a_{i j}$ for $i \neq j$. Meanwhile, a matrix $L$ is called symmetric if its corresponding undirected graph has symmetric weights, that is, $a_{i j}=a_{j i}$. In a directed graph, a directed path is a sequence of edges $\left(v_{1}, v_{2}\right),\left(v_{2}, v_{3}\right), \ldots$, where $v_{i} \in V$. A directed graph has a directed spanning tree if at least one node has a directed path to all other nodes. A strongly connected graph means that each node has a directed path to all other nodes. In this paper, for convenience, we suppose that the weight of all edges is 1 and graphs are undirected.

The community detection algorithm can be referred to the following idea based on [23]: The agent with the highest degree of importance will be selected as the cluster point of community in each iteration; the agent included in community neighbor set and making the largest increase for local module degree should be contained in community; next, the neighbor set and local modularity of community are further updated. The new community is formed until the local modularity does not increase or neighbor set is null.

Lemma 2.1 ([4]) (i) All the eigenvalues of the Laplacian matrix $L$ have nonnegative real parts; (ii) Zero is an eigenvalue of $L$ with $1_{n}$ (where $1_{n}$ is the $n \times 1$ column vector of all ones) as the corresponding right eigenvector. Furthermore, zero is a simple eigenvalue of $L$ if and only if the graph $G$ has a directed spanning tree.

\section{The average consensus of first-order multiagent systems with FCWAC Algorithm}

To obtain fast average consensus, we present FCWAC Algorithm. We will provide it further.

\subsection{Fast convergent weighted average consensus algorithm-FCWAC Algorithm}

The first step: according to the community detection algorithm [23], we divide the communication topology network into several communities.

The second step: let every community to reach consensus (information exchange only among the internal agents of every community). 
The third step: let every community be an agent. Finally, the new system reaches the weighted average consensus.

According to the FCWAC Algorithm, multiagent systems can reach average consensus. We will further analyze the reason in Remark 3.1.

\subsection{The weighted average consensus of first-order multiagent systems}

Let $m$ denote the number of agents, $n$ denote the number of communities, and $n_{i}$ denote the number of agents in the community $i=1,2, \ldots, n$. We provide the following dynamics equations:

$$
w_{i} \dot{x}_{i}(t)=u_{i}(t), \quad i=1,2, \ldots, n,
$$

where $x_{i}(t)$ and $u_{i}(t)$ denote the position and control input of agent $i$, respectively. The consensus protocol $u_{i}(t)$ is given as

$$
u_{i}(t)=\sum_{j \in \mathcal{N}_{i}} a_{i j}\left(x_{j}(t)-x_{i}(t)\right)
$$

where $a_{i j} \geq 0, W=\operatorname{Diag}\left(w_{1}, w_{2}, \ldots, w_{n}\right), w_{i}=\frac{n_{i}}{m}, n_{i} \neq 0$.

Furthermore, these equations can be rewritten in the vector form:

$$
\dot{x}(t)=-W^{-1} L x(t) .
$$

For the multiagent system (3), we have the following result.

Theorem 3.1 Let $G$ be a strongly connected graph. Then under protocol (2) system (1) (every community is seen as an agent) can reach the weighted average consensus, and

$$
x(t)=1_{n} \frac{1_{n}^{T} W}{\left\|1_{n}^{T} W\right\|_{1}} x(0)
$$

where $1_{n}$ is the vector of all ones.

Proof In terms of Lemma 2.1, $L$ has a simple zero eigenvalue. Because all the diagonal entries of $W$ are positive, $W$ is also an invertible matrix. For the Laplacian matrix $L$, all the Getschgorin disks lie at the right region of the $y$-axis of the rectangular plane coordinate system. Thus all the eigenvalues of $L$ are positive except 0 . Next, let us multiply the matrix $L$ by $W^{-1}$ from the left side of $L$. Thus these Getschgorin disks still lie at the right region of the $y$-axis. So the eigenvalues of $W^{-1} L$ are also positive except 0 . Moreover, $W^{-1} L$ also has a simple zero eigenvalue. Thus system (3) is asymptotically stable. Obviously, we can obtain that $1_{n}$ is a right eigenvector of $W^{-1} L$ corresponding to the zero eigenvalue, and $1_{n}^{T} W$ is a left eigenvector of $W^{-1} L$ corresponding to the zero eigenvalue.

Meanwhile, $W^{-1} L$ can be rewritten as the Jordan canonical form $S J S^{-1}$, where $J$ is the Jordan block matrix with its eigenvalues being the diagonal entries. For the columns of $S$, $s_{k}, k=1, \ldots, n$, can be chosen to be the right eigenvectors or generalized right eigenvectors associated with eigenvalues of $W^{-1} L$. Similarly, $h_{k}^{T}, k=1, \ldots, n$, which are the rows of $S^{-1}$ satisfying $h_{k}^{T} s_{k}=1, h_{k}^{T} s_{l}=0(k \neq l)$, can be chosen to be the left eigenvectors or generalized 
left eigenvectors associated with eigenvalues of $W^{-1} L$. Furthermore, we choose $s_{1}=1_{n}$, $h_{1}^{T}=\frac{1_{n}^{T} W}{\left\|1_{n}^{T} W\right\|_{1}}$ as the eigenvectors corresponding to the zero eigenvalue.

So we have

$$
\begin{aligned}
\lim _{t \rightarrow \infty} x(t) & =e^{-W^{-1} L t} x(0) \\
& =\left(\begin{array}{lll}
s_{1} & \cdots & s_{n}
\end{array}\right) \cdot\left(\begin{array}{cc}
1 & 0_{1 \times(n-1)} \\
0_{(n-1) \times 1} & 0_{(n-1) \times(n-1)}
\end{array}\right) \cdot\left(\begin{array}{c}
h_{1}^{T} \\
\vdots \\
h_{n}^{T}
\end{array}\right) x(0) \\
& =1_{n} h_{1}^{T} x(0)=1_{n} \frac{1_{n}^{T} W}{\left\|1_{n}^{T} W\right\|_{1}} x(0),
\end{aligned}
$$

which implies $x_{i}(t) \rightarrow \frac{1_{n}^{T} W}{\left\|1_{n}^{T} W\right\|_{1}} x(0), i=1,2, \ldots, n$. Therefore the weighted average consensus can be obtained.

Remark 3.1 In terms of the third step of our FCWAC Algorithm, every community is considered as an agent (the subsystem formed by one single community has already reached consensus, and every agent in the same community has the same state). Therefore we have $n_{i}$ agents with the same state in the community $i$. Due to $w_{i}=\frac{n_{i}}{m}, i=1,2, \ldots, n$, we can easily obtain $\frac{1_{n}^{T} W}{\left\|1_{n}^{T} W\right\|_{1}} x(0)=1_{n}^{T} x^{\prime}(0)$, where $x(0)$ is the initial condition of agents in the third step of the FCWAC Algorithm, and $x^{\prime}(0)$ is the initial condition of the second step in the FCWAC Algorithm. Therefore our algorithm can indeed reach average consensus.

\section{The average consensus of second- and high-order multiagent systems using the FCWAC Algorithm}

For the average consensus of second- and high-order systems, we still use our FCWAC algorithm to process it. The weighted consensus of second-order multiagent systems is considered in the following section.

\subsection{The average consensus of second-order multiagent systems using the FCWAC Algorithm}

Similarly, let $m$ still denote the number of agents, $n$ denote the number of communities, and $n_{i}$ denote the number of agents in the community $i=1,2, \ldots, n$. We present the following dynamics equations:

$$
\begin{aligned}
& w_{i} \dot{x}_{i}(t)=v_{i}(t), \quad i=1,2, \ldots, n, \\
& w_{i} \dot{v}_{i}(t)=u_{i}(t), \quad i=1,2, \ldots, n,
\end{aligned}
$$

where $x_{i}(t), v_{i}(t)$, and $u_{i}(t)$ denote the position, velocity, and control input of agent $i$, respectively. The consensus protocol $u_{i}(t)$ is given as

$$
u_{i}(t)=\sum_{j \in \mathcal{N}_{i}} a_{i j}\left(x_{j}(t)-x_{i}(t)\right)+\sum_{j \in \mathcal{N}_{i}} a_{i j}\left(v_{j}(t)-v_{i}(t)\right),
$$

where $a_{i j} \geq 0, W=\operatorname{Diag}\left(w_{1}, w_{2}, \ldots, w_{n}\right), w_{i}=\frac{n_{i}}{m}$. 
Furthermore, these equations can be rewritten in the vector form:

$$
\left(\begin{array}{l}
x(t) \\
v(t)
\end{array}\right)=\Phi\left(\begin{array}{l}
x(t) \\
v(t)
\end{array}\right)
$$

where $\Phi=\left(\begin{array}{cc}0 & W^{-1} \\ -W^{-1} L & -W^{-1} L\end{array}\right)$.

For system (6), we have the following result.

Theorem 4.1 Let $G$ be a strongly connected graph. If (8) has only real roots or all the nonreal complex roots of (8) have negative real parts, then, under protocol (6), system (7) (every community is seen as an agent) can reach the weighted average consensus given by

$$
\left(\begin{array}{l}
x(t) \\
v(t)
\end{array}\right)=\left(\begin{array}{c}
1_{n} \frac{1_{n}^{T} W}{\left\|1_{n}^{T} W\right\|_{1}} x(0)+t 1_{n} \frac{1_{n}^{T} W}{\left\|1_{n}^{T} W\right\|_{1}} v(0) \\
1_{n} \frac{1_{n}^{T} W}{\left\|1_{n}^{T} W\right\|_{1}} v(0)
\end{array}\right) .
$$

Proof According to the characteristic equation

$$
\left(\begin{array}{cc}
0 & W^{-1} \\
-W^{-1} L & -W^{-1} L
\end{array}\right)\left(\begin{array}{l}
x(t) \\
v(t)
\end{array}\right)=\lambda\left(\begin{array}{l}
x(t) \\
v(t)
\end{array}\right),
$$

we get

$$
\left(W^{-1} L W^{-1}+\lambda W^{-1} L+\lambda^{2} I_{n}\right) W x(t)=0 .
$$

To obtain the eigenvalues of $\Phi$, we only need to solve

$$
\left|L+\lambda L W+\lambda^{2} W^{2}\right|=0
$$

Further, we only need to solve

$$
\left|L\left(I_{n}+\lambda W\right)+\lambda^{2} W^{2}\right|=0
$$

If $\lambda \in R$ and $\lambda>0$, then we get

$$
\left|\left(I_{n}+\lambda W\right)^{T}\right|\left|L\left(I_{n}+\lambda W\right)+\lambda^{2} W^{2}\right|=\left|\left(I_{n}+\lambda W\right)^{T} L\left(I_{n}+\lambda W\right)+\lambda^{2}\left(I_{n}+\lambda W\right)^{T} W^{2}\right| \neq 0 .
$$

So $\left|L\left(I_{n}+\lambda W\right)+\lambda^{2} W^{2}\right| \neq 0$. Furthermore, we can get that the real eigenvalues of $\Phi$ are not positive.

From (8) we can also obtain that 0 is an eigenvalue of $\Phi$ and its algebraic multiplicity is 2 .

If (8) has nonreal complex roots or negative real roots and if all nonreal complex eigenvalues of (8) have negative real parts, then we can prove that e system (7) can reach consensus, which will be proved in the following paragraph.

Furthermore, $\Phi$ can also be written as the Jordan canonical form $S J S^{-1}$, where the columns of $S$, denoted by $s_{k}, k=1, \ldots, 2 n$, can be chosen to be the right eigenvectors or generalized right eigenvectors associated with eigenvalues of $\Phi ; h_{k}^{T}, k=1, \ldots, 2 n$, which are 
the rows of $S^{-1}$ and satisfy $h_{k}^{T} s_{k}=1, h_{k}^{T} s_{l}=0(k \neq l)$, can be chosen to be the left eigenvectors or generalized left eigenvectors of $\Phi$, and $J$ is the Jordan block matrix with its eigenvalues being the diagonal entries. We get that $s_{1}=\left(\begin{array}{c}1_{n} \\ 0\end{array}\right), s_{2}=\left(\begin{array}{c}0 \\ 1_{n}\end{array}\right)$ are the right eigenvectors or the generalized right eigenvectors, $h_{1}^{T}=\frac{1}{\left\|1_{n}^{T} W^{-1}\right\|_{1}}\left(\begin{array}{c}1_{n}^{T} \\ 0\end{array}\right)^{T} W^{-1}$ and $h_{2}^{T}=\frac{1}{\left\|1_{n}^{T} W^{-1}\right\|_{1}}\left(\begin{array}{c}0 \\ 1_{n}^{T}\end{array}\right) W^{-1}$ are the left eigenvectors or the generalized left eigenvectors corresponding to the Jordan block $\left(\begin{array}{ll}0 & 1 \\ 0 & 0\end{array}\right)$. Thus we obtain

$$
\begin{aligned}
\lim _{t \rightarrow \infty}\left(\begin{array}{l}
x(t) \\
v(t)
\end{array}\right) & =e^{\Phi t}\left(\begin{array}{l}
x(0) \\
v(0)
\end{array}\right) \\
& =\left(\begin{array}{lll}
s_{1} & \cdots & s_{2 n}
\end{array}\right) \cdot\left(\begin{array}{cc}
\left(\begin{array}{cc}
1 \\
0 & 1
\end{array}\right) & 0_{2 \times(2 n-2)} \\
0(2 n-2) \times 2 & 0_{(2 n-2) \times(2 n-2)}
\end{array}\right) \cdot\left(\begin{array}{c}
h_{1}^{T} \\
\vdots \\
h_{2 n}^{T}
\end{array}\right)\left(\begin{array}{c}
x(0) \\
v(0)
\end{array}\right) \\
& =\left(s_{1} h_{1}^{T}+t s_{1} h_{2}^{T}+s_{2} h_{2}^{T}\right)\left(\begin{array}{c}
x(0) \\
v(0)
\end{array}\right)=\left(\begin{array}{c}
\frac{1_{n} n_{n}^{T} W^{-1} x(0)}{\left\|1_{n}^{T} W^{-1}\right\|_{1}}+t \frac{1_{n} 1_{n}^{T} W^{-1} v(0)}{\left\|11_{n}^{T} W^{-1}\right\|_{1}} \\
\frac{1_{n} 1_{n}^{T} W^{-1} v(0)}{\left\|1{ }_{n}^{T} W^{-1}\right\|_{1}}
\end{array}\right),
\end{aligned}
$$

which implies that $x_{i}(t) \rightarrow \frac{1_{n}^{T} W^{-1} x(0)}{\left\|1_{n}^{T} W^{-1}\right\|_{1}}+t \frac{1_{n}^{T} W^{-1} v(0)}{\left\|1_{n}^{T} W^{-1}\right\|_{1}}$ and $v_{i}(t) \rightarrow \frac{1_{n}^{T} W^{-1} v(0)}{\left\|1_{n}^{T} W^{-1}\right\|_{1}}$. So the weighted average consensus can be obtained.

Remark 4.1 According to our FCWAC Algorithm, the second-order multiagent system can reach the average consensus. The reason is similar to the explanation in Remark 3.1.

\subsection{Fast average consensus of high-order multiagent systems}

In the following part, we consider a high-order weighted dynamical consensus of multiagent system.

Let $m$ denote the number of agents, $n$ denote the number of communities, and $n_{i}$ denote the number of agents in the community $i=1,2, \ldots, n$. Consider the multiagent systems with $l$ th-order dynamics $(l \geq 3)$ given by

$$
\begin{aligned}
& w_{i} \dot{x}_{i}(t)=x_{i}^{(1)}(t), \\
& \vdots \\
& w_{i} \dot{x}_{i}^{(l-2)}(t)=x_{i}^{(l-1)}(t), \\
& w_{i} \dot{x}_{i}^{(l-1)}(t)=u_{i}(t),
\end{aligned}
$$

where $x_{i}^{(d)}(t), d=0, \ldots, l-1$, are the states of agents, $u_{i}(t)$ is the control input, and $x_{i}^{(d)}(t)$ denotes the $k$ th derivative of $x_{i}(t)$ with $x_{i}^{(0)}(t)=x_{i}(t), i=1, \ldots, n$. Meanwhile, $a_{i j} \geq 0, W=$ $\operatorname{Diag}\left(w_{1}, w_{2}, \ldots, w_{n}\right), w_{i}=\frac{n_{i}}{m}$. Next, we consider the following dynamical protocol:

$$
u_{i}(t)=\sum_{d=0}^{l-1} \sum_{j \in \mathcal{N}_{i}} a_{i j}(t)\left(x_{j}^{(d)}(t)-x_{i}^{(d)}(t)\right) .
$$


Furthermore, we can rewritten these equations as follows:

$$
\begin{aligned}
& W \dot{x}(t)=x^{(1)}(t), \\
& \vdots \\
& W \dot{x}^{(l-2)}(t)=x^{(l-1)}(t), \\
& W \dot{x}^{(l-1)}(t)=-\sum_{d=o}^{l-1} L x^{(d)}(t) .
\end{aligned}
$$

Finally, they can be rewritten as

$$
\dot{X}(t)=\Psi X(t),
$$

where $X(t)=\left(x(t) x^{(1)}(t) \cdots x^{(l-1)}(t)\right)^{T}$ and

$$
\Psi=\left(\begin{array}{ccccc}
0 & I & 0 & \cdots & 0 \\
0 & 0 & I & \cdots & 0 \\
0 & 0 & 0 & \cdots & 0 \\
\cdots & \cdots & \cdots & \cdots & \cdots \\
-W^{-1} L & -W^{-1} L & -W^{-1} L & \cdots & -W^{-1} L
\end{array}\right) .
$$

For high-order multiagent systems, similarly, we have the following theorem.

Theorem 4.2 Let $G$ be a strongly connected graph. If $\Psi$ has only real eigenvalues or all the nonreal complex eigenvalues of $\Psi$ have negative real parts, then, under protocol (6), system (11) (every community is seen as an agent) can reach the weighted average consensus given by

$$
\left(\begin{array}{c}
x(t) \\
x^{(1)}(t) \\
\cdots \\
x^{(l-1)}(t)
\end{array}\right)=\left(\begin{array}{c}
1_{n} \frac{1_{n}^{T} W}{\left\|1_{n}^{T} W\right\|_{1}} x(0)+\cdots+\frac{t^{l-1}}{(l-1) !} 1_{n} \frac{1_{n}^{T} W}{\left\|1_{n}^{T} W\right\|_{1}} x^{l-1}(0) \\
1_{n} \frac{1_{n}^{T} W}{\left\|1_{n}^{T} W\right\|_{1}} x^{1}(0)+\cdots+\frac{t^{l-2}}{(l-2) !} 1_{n} \frac{1_{n}^{T} W}{\left\|1_{n}^{T} W\right\|_{1}} x^{l-1}(0) \\
\cdots \\
1_{n} \frac{1_{n}^{T} W}{\left\|1_{n}^{T} W\right\|_{1}} x^{l-1}(0)
\end{array}\right) .
$$

Remark 4.2 According to our FCWAC Algorithm, the high-order multiagent system still can reach average consensus.

\section{Simulation}

In this section, we present two simulation examples to illustrate our consensus protocols. In the following examples, we consider a multiagent system consisting of 20 agents with topology in Fig. 1 and initial condition $x(0)=(0.2,0.3,0.5,0.7,0.9,1.1,1.3,1.5,1.7,1.8,2.1$, $2.3,2.5,3.1,3.2,3.3,3.4,3.6,3.7,3.9)^{T}$. For comparison, we choose the same topology graph of the example in [23]. The multiagent system is divided into four communities: community 1 [1-5], community 2 [6-8], community 3 [9-13], and community 4 [14-20]. Thus the weighted matrix is equal to $\operatorname{Diag}(1 / 4,3 / 20,1 / 4,7 / 20)$. The Laplacian matrices of each 


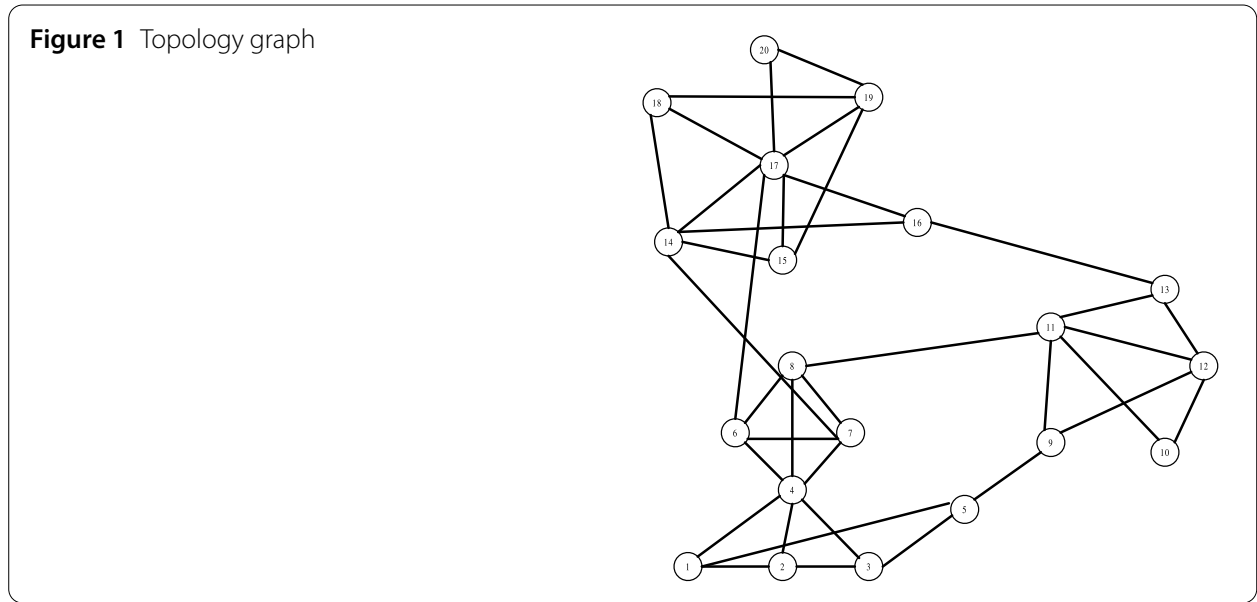

community are

$$
\begin{aligned}
L_{1} & =\left(\begin{array}{ccccc}
3 & -1 & 0 & -1 & -1 \\
-1 & 3 & -1 & -1 & 0 \\
0 & -1 & 3 & -1 & -1 \\
-1 & -1 & -1 & 3 & 0 \\
-1 & 0 & -1 & 0 & 2
\end{array}\right), \quad L_{2}=\left(\begin{array}{ccc}
2 & -1 & -1 \\
-1 & 2 & -1 \\
-1 & -1 & 2
\end{array}\right) \\
L_{3} & =\left(\begin{array}{ccccc}
2 & 0 & -1 & -1 & 0 \\
0 & 2 & -1 & -1 & 0 \\
-1 & -1 & 4 & -1 & -1 \\
-1 & -1 & -1 & 4 & -1 \\
0 & 0 & -1 & -1 & 2
\end{array}\right), \text { and } \\
L_{4} & =\left(\begin{array}{ccccccc}
4 & -1 & -1 & -1 & -1 & 0 & 0 \\
-1 & 3 & 0 & -1 & 0 & -1 & 0 \\
-1 & 0 & 2 & -1 & 0 & 0 & 0 \\
-1 & -1 & -1 & 6 & -1 & -1 & -1 \\
-1 & 0 & 0 & -1 & 3 & -1 & 0 \\
0 & -1 & 0 & -1 & -1 & 4 & -1 \\
0 & 0 & 0 & -1 & 0 & -1 & 2
\end{array}\right),
\end{aligned}
$$

respectively. In the third step of the FCWAC Algorithm, the weighted Laplacian matrix is equal to

$$
L_{5}=\left(\begin{array}{cccc}
4 & -3 & -1 & 0 \\
-3 & 6 & -1 & -2 \\
-1 & -1 & 3 & -1 \\
0 & -2 & -1 & 3
\end{array}\right) .
$$

Example 5.1 In this example, we consider the consensus of the first-order multiagent system. Figure 2(a) is the state trajectory of the multiagent system without community division. Figure 2(b) is the state trajectory of the multiagent system using the community 


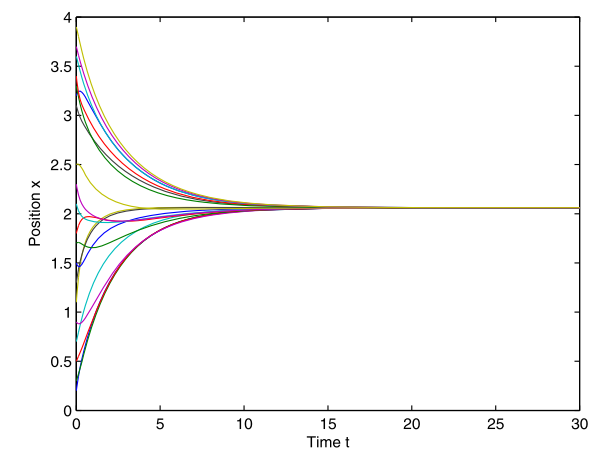

(a) Agent states without community division

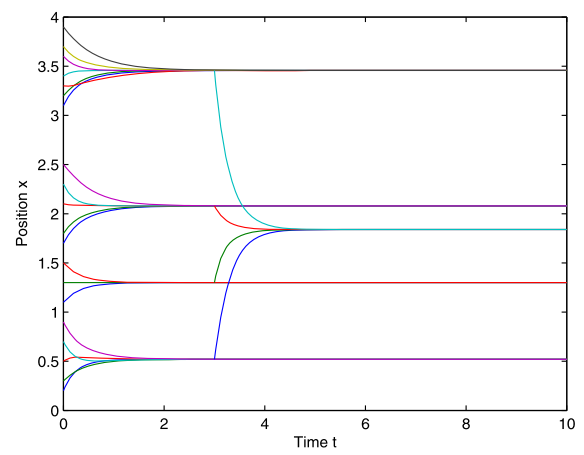

(b) Agent states using algorithm in [11]

Figure 2 Agent states of multiagent system

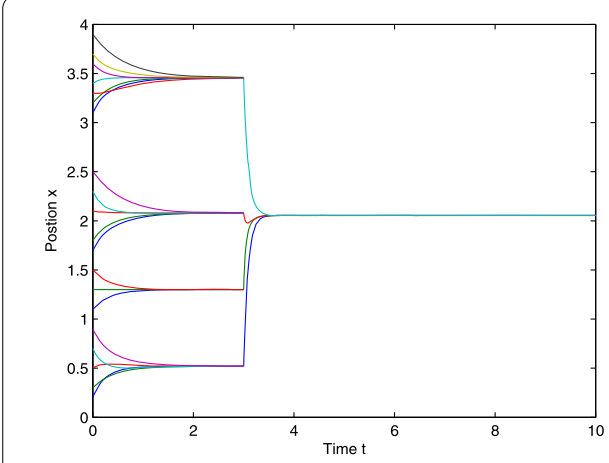

(a) Agents Position using FCWAC Algorithm

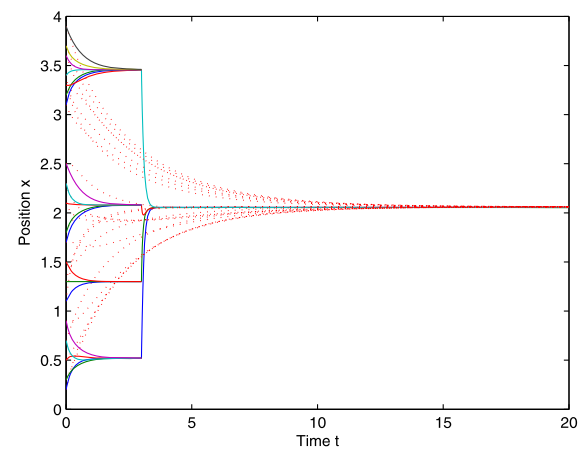

(b) Agents Position comparison

Figure 3 State trajectories of multiagent system

detection algorithm in [23]. Obviously, from Fig. 2(b) we can find that the multiagent system do not obtain average consensus.

Figure 3(a) is the state trajectory of the multiagent system using the FCWAC Algorithm. We can find that the multiagent system obtains faster convergence speed than it in the case without division community and indeed reaches the average consensus. Figure 3(b) is a comparison figure of state trajectory between using the FCWAC Algorithm and without division community case. Moreover, we can find that our FCWAC Algorithm has faster convergence speed than the community detection algorithm in [23]. We also find that the final states of the agents verify the correctness of Theorem 3.1.

Example 5.2 In this example, we consider the consensus of second-order multiagent system. Figure 4 is the state trajectory of the multiagent system without community division. Figure 5 is the state trajectory of the multiagent system using the FCWAC Algorithm. We can find that the multiagent system obtains faster convergence speed than it in the case without division community, and the multiagent system indeed reaches the average consensus. Figure 6 is a comparison figure of state trajectory between using the FCWAC Algorithm and without division community case. The final states of the agents verify the correctness of Theorem 4.1. 


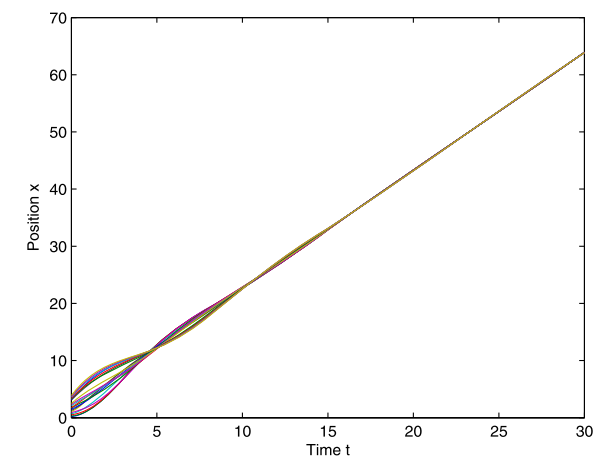

(a) Position without community division

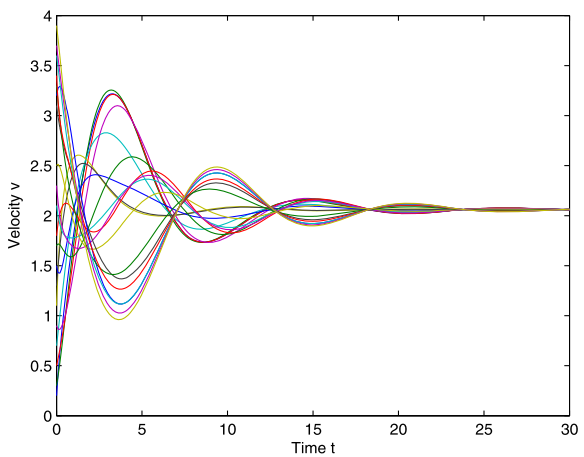

(b) Velocity without community division

Figure 4 State trajectories of multiagent system

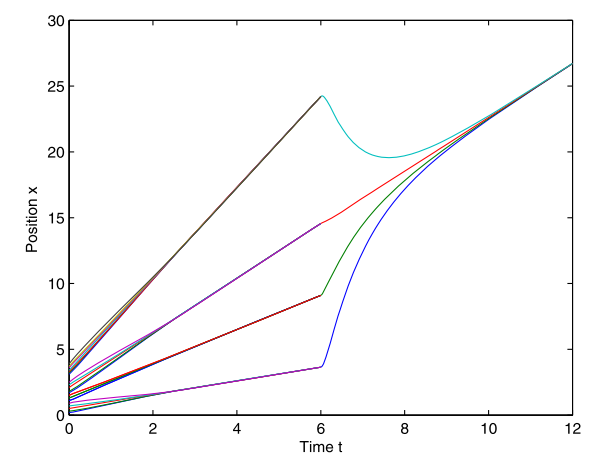

(a) Agents position using FCWAC Algorithm

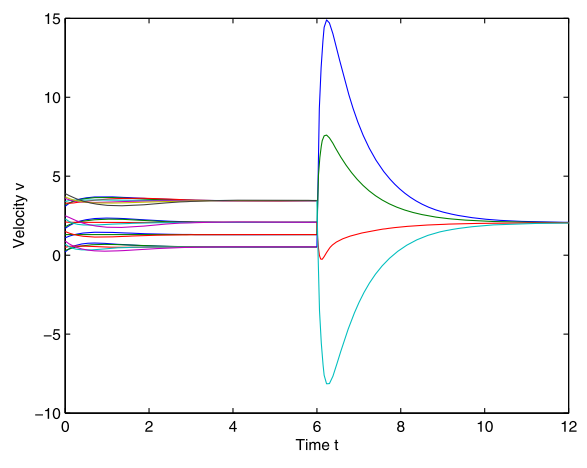

(b) Velocity using FCWAC Algorithm

Figure 5 State trajectories of multiagent system

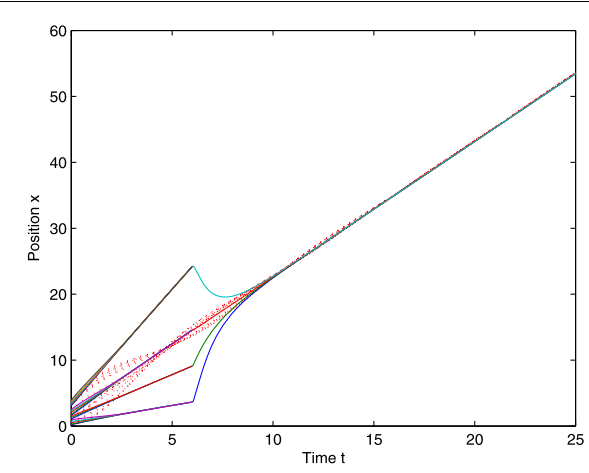

(a) Position comparison trajectories

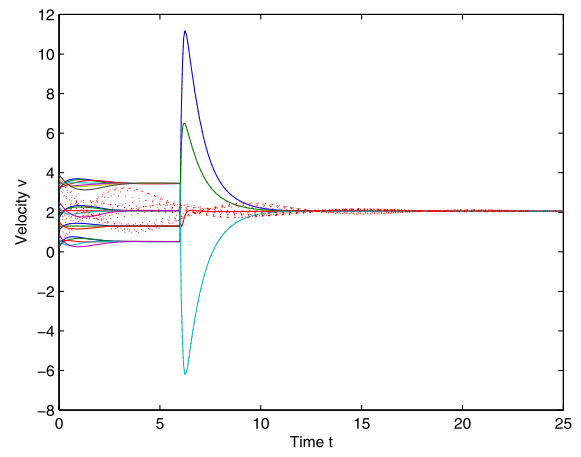

(b) Velocity comparison trajectories

Figure 6 State trajectories of multiagent system

\section{Conclusion}

In this paper, we consider fast convergent average consensus based on community detection algorithm and present the FCWAC Algorithm. To use the FCWAC Algorithm, we obtain the results of the weighted average consensus of multiagent systems. It should be 
noticed that our idea can also solve the average consensus problem, which divides the communication topology into several layers of community. Finally, we use numerical examples to illustrate our theoretical results.

\section{Acknowledgements}

The authors are grateful to editors and reviewers for their valuable suggestions.

\section{Funding}

This work is part supported by China Scholarship council (2014085151114), Scientific Research Fund of Sichuan Provincial Education Department (16ZA0304), and Research Project of Leshan Normal University (Z1514).

\section{Competing interests}

The authors declare that they have no competing interests.

\section{Authors' contributions}

The authors declare that the study was realized in collaboration with the same responsibility. All authors read and approved the final manuscript.

\section{Author details}

${ }^{1}$ College of Mathematics and Information Science, Leshan Normal University, Leshan, China. ${ }^{2}$ Department of Math/Stat, Georgia State University, Atlanta, USA. ${ }^{3}$ College of the Special Education, Leshan Normal University, Leshan, China.

\section{Publisher's Note}

Springer Nature remains neutral with regard to jurisdictional claims in published maps and institutional affiliations.

Received: 18 June 2018 Accepted: 21 November 2018 Published online: 28 November 2018

\section{References}

1. Borkar, V., Varaiya, P.: Asymptotic agreement in distributed estimation. IEEE Trans. Autom. Control 27(3), 650-655 (1982)

2. Tsitsiklis, J.: Problems in decentralized decision making and computation. Dissertation, Massachusetts Institute of Technology (1984)

3. Vicsek, T., Cziroo, A., Ben-Jacob, E., Cohen, I., Shochet, O.: Novel type of phase transition in a system of self-driven particles. Phys. Rev. Lett. 75(6), 1226-1229 (1995)

4. Olfati-Saber, R., Murray, R.: Consensus problems in the networks of agents with switching topology and time delays. IEEE Trans. Autom. Control 49(9), 1520-1533 (2004)

5. Xiao, L., Boyd, S.: Fast linear iterations for distributed averaging. Syst. Control Lett. 53(1), 65-78 (2004)

6. Xiao, L., Boyd, S., Kim, S.J.: Distributed average consensus with least-mean-square deviation. J. Parallel Distrib. Comput. 67(1), 33-46 (2007)

7. Kokiopoulou, E., Frossard, P.: Polynomial filtering for fast convergence in distributed consensus. IEEE Trans. Signal Process. 57(1), 342-354 (2009)

8. Li, J., Xu, S., Chu, Y., Wang, H.: Distributed average consensus control in networks of agents using outdated states. IET Control Theory Appl. 4(5), 746-758 (2010)

9. Aysal, T., Oreshkin, B., Coates, M.: Accelerated distributed average consensus via localized node state prediction. IEEE Trans. Signal Process. 57(4), 1563-1576 (2009)

10. Oreshkin, B., Coates, M., Rabbat, M.: Optimization and analysis of distributed averaging with short node memory. IEEE Trans. Signal Process. 58(5), 2850-2865 (2010)

11. Lin, P., Jia, Y.: Average consensus in networks of multi-agents with both switching topology and coupling time-delay. Physica A 387, 303-313 (2008)

12. Zhu, J., Tian, Y., Kuang, J.: On the general consensus protocol of multi-agent systems with double integrator dynamics. Linear Algebra Appl. 431, 701-715 (2009)

13. Ren, W.: Collective motion from consensus with Cartesian coordinate coupling. Part II: double-integrator dynamics. In: Proceedings of the 47th IEEE Conference on Decision and Control, Mexico, pp. 1012-1017 (2008)

14. Su, H., Wang, X.: Second-order consensus of multiple agents with coupling delay. In: Proceedings of the 7th World Congress on Intelligent Control and Automation, pp. 7181-7186. IEEE, Chongqing (2008)

15. Zhang, W., Zeng, D., Qu, S.: Dynamic feedback consensus control of a class of high-order multi-agent systems. IET Control Theory Appl. 4(10), 2219-2222 (2010)

16. Rezaee, H., Abdollahi, F.: Discrete-time consensus strategy for a class of high-order linear multiagent systems under stochastic communication topologies. J. Franklin Inst. 354, 3690-3705 (2017)

17. Huang, J., Fang, H., Dou, L., Chen, J.: An overview of distributed high-order multi-agent coordination. IEEE/CAA J. Autom. Sin. 1(1), 1-9 (2014)

18. Lin, P., Ren, W., Yang, C., Gui, W.: Distributed consensus of second-order multiagent systems with nonconvex velocity and control input constraints. IEEE Trans. Autom. Control 63(4), 1171-1176 (2018)

19. Xu, X., Liu, L., Feng, G.: Consensus of discrete-time linear multiagent systems with communication, input and output delays. IEEE Trans. Autom. Control 63(2), 492-497 (2018)

20. $\mathrm{Wu}, \mathrm{Z} ., \mathrm{Xu}, \mathrm{Y} ., \mathrm{Lu}, \mathrm{R}$., Wu, Y., Huang, T.: Event-triggered control for consensus of multiagent systems with fixed/switching topologies. IEEE Trans. Syst. Man Cybern. Syst. 48(10), 1736-1746 (2018)

21. Zhang, J., Zhang, H., Feng, T.: Distributed optimal consensus control for nonlinear multiagent system with unknown dynamic. IEEE Trans. Neural Netw. Learn. Syst. 29(8), 3339-3348 (2018) 
22. Rehan, M., Jameel, A., Ahn, C.: Distributed consensus control of one-sided Lipschitz nonlinear multiagent systems. IEEE Trans. Syst. Man Cybern. Syst. 48(10), 1297-1308 (2018)

23. Zhang, B., Chang, D., Li, Z., Ma, D.: On convergence rate for multi-agent consensus: a community detection algorithm. In: 32nd Youth Academic Annual Conference of Chinese Association of Automation, pp. 72-77. IEEE, Hefei (2017)

24. Asmi, K., Lotfi, D., Marraki, M.: An approach based on the clustering coefficient for the community detection in social networks. In: International Conference on Security of Smart Cities, vol. 61, pp. 1-8. IEEE, Paris (2016)

25. Epstein, M., Lynch, K., Johansson, K., Murray, R.: Using hierarchical decomposition to speed up average consensus. In: Proceedings of International Federation of Automatic Control (IFAC) World Congress, pp. 612-618. IFEE, Seoul (2008)

26. Li, X., Xi, Y: Hierarchically decomposing multi-agent system to accelerate group consensus. In: Proceedings of Asian Control Conference, pp. 347-352. IEEE, Kaohsiung (2011)

\section{Submit your manuscript to a SpringerOpen ${ }^{\circ}$ journal and benefit from:}

- Convenient online submission

- Rigorous peer review

- Open access: articles freely available online

High visibility within the field

Retaining the copyright to your article

Submit your next manuscript at $\gg$ springeropen.com 